\title{
Poly-Left-Lactic Acid Tubular Scaffolds via Diffusion Induced Phase Separation: Control of Morphology
}

\author{
Francesco Carfi Pavia, ${ }^{1}$ Vincenzo La Carrubba, ${ }^{2}$ Giulio Ghersi, ${ }^{3}$ Valerio Brucato ${ }^{1}$ \\ ${ }^{1}$ Dipartimento di Ingegneria Chimica, Gestionale, Informatica, Meccanica, Università di Palermo, Palermo, Italy \\ ${ }^{2}$ Dipartimento di Ingegneria Civile, Ambientale, Aerospaziale, dei Materiali, Università di Palermo, \\ Palermo, Italy \\ ${ }^{3}$ Dipartimento di Scienze e Tecnologie Molecolari e Biomolecolari, Università di Palermo, Palermo, Italy
}

\begin{abstract}
In this work, tubular poly-left-lactic acid scaffolds for vascular tissue engineering applications were produced by an innovative two-step method. The scaffolds were obtained by performing a dip-coating around a nylon fiber, followed by a diffusion induced phase separation process. Morphological analysis revealed that the internal lumen of the as-obtained scaffold is equal to the diameter of the fiber utilized; the internal surface is homogeneous with micropores 1-2 $\mu \mathrm{m}$ large. Moreover, a porous open structure was detected across the thickness of the walls of the scaffold. An accurate analysis of the preparation process revealed that it is possible to tune up the morphology of the scaffold (wall thickness, porosity, and average pore dimension), simply by varying some experimental parameters. Preliminary in vitro cell culture tests were carried out inside the scaffold. The results showed that cells are able to grow within the internal surface of the scaffolds and after 3 weeks they begin to form a "primordial" vessel-like structure. Modeling predictions of the dipcoating process display always an underestimate of experimental data (dependence of wall thickness upon extraction rate). POLYM. ENG. SCI., 00:000-000, 2012. (C) 2012 Society of Plastics Engineers
\end{abstract}

\section{INTRODUCTION}

A great deal of research has been pursued in the last decade with the goal of developing blood vessel substitutes, owing to the large impact this advance would have on the millions of patients that annually suffer from diseases of blood vessels [1]. Strategies to engineer blood vessel must provide adequate mechanical properties, to avoid catastrophic failure in this mechanically demanding

Correspondence to: Vincenzo La Carrubba;e-mail: vincenzo.lacarruba@ unipa.it

DOI 10.1002/pen.23273

Published online in Wiley Online Library (wileyonlinelibrary.com).

(C) 2012 Society of Plastics Engineers site, and appropriate cellular components to form the complex vascular wall.

Despite a clear clinical need for a functional arterial graft, success has been limited to arterial replacements of large-caliber vessels such as, the thoracic and abdominal aorta, arch vessels, iliac, and common femoral arteries. Currently, occluded vessels with diameters below $6 \mathrm{~mm}$ are by passed with autologs native blood vessels, such as the saphenus vein. However, those surgical techniques, which account for a majority of the demand, have generally proved inadequate, largely because of acute thrombogenicity of the graft, anastomotic intimal hyperplasia, aneurysm formation, infection, and progression of atherosclerotic disease [2].

Tissue engineering has emerged as a promising approach to address the shortcomings of current options. Investigators have explored the use of arterial tissue cells combined with various types of natural and synthetic scaffolds to make tubular constructs and subject them to chemical and/or mechanical stimulation in an attempt to develop a functional small-diameter arterial replacement graft with varying degrees of success [3].

The general concept for creating a tissue-engineered vascular graft (TEVG) usually involves the harvest of desired cells, cell expansion in culture, cell seeding onto a scaffold; construct culture in an environment that induces tissue formation, and implantation of the construct back into the patient [4].

The grafts must be biocompatible in several aspects: nonthrombogenic, noninflammatory, and nonimmunogenic. Moreover, grafts must closely mimic the unique viscoelastic nature of an artery (this is particularly important for small-diameter arteries), must accommodate pressure changes, and be nondisruptive to blood flow. Finally, they should also be able to "remodel" efficiently, i.e., to allow for growth of a layer of endothelium in a reasonable period of time, compatibly with the biodegradation 
TABLE 1. Thickness of synthetic blood vessels prepared according to procedures reported in literature.

\begin{tabular}{lccc}
\hline & & & $\begin{array}{c}\text { Wall } \\
\text { thickness } \\
(\mu \mathrm{m})\end{array}$ \\
\hline Suthors & Technique & Material & 390 \\
Nieponice et al. [27] & TIPS/electrospinning & PEUU & 300 \\
Roh et al. [28] & TIPS & PEUU & 150 \\
Song et al. [29] & Molding & PLLA/PLGA & 900 \\
& Dip coating/ & PTMC & \\
\hline
\end{tabular}

kinetics of the graft itself, when constituted by a polymer that overcomes a hydrolytic degradation [5].

Traditionally, the most widely employed non-degradable materials include Dacron ${ }^{\mathrm{TM}}$ (polyethyleneterepthalate; PET), Gore-Tex ${ }^{\mathrm{TM}}$ (expanded polytetrafluoroethylene; ePTFE), and compliant polyurethanes. Unfortunately, these polymers have been used successfully in peripheral revascularization but failed in coronary revascularization [6]. Dacron grafts lead to thrombosis and neointimal thickening in low blood flow. The ePTFE grafts also fail owing to surface thrombogenicity for small vessels [7]. Conversely, several biodegradable polymers such as polyglycolic acid [8, 9], polylactic acid, poly( $\varepsilon$-caprolactone) [10-13], and polyurethane [14] have been investigated to serve as vascular scaffolds. In particular, poly-left-lactic acid (PLLA) and the other polylactides are organic polymers used frequently in the preparation of porous supports, mainly due to their very good biocompatibility. As far as the biodegradation of the PLLA is concerned, two different modes are defined, namely (1) hydrolytic degradation or hydrolysis, which is mediated simply by water and (2) enzymatic degradation, which is mainly mediated by biological agents such as enzymes. However, the rate of scaffold degradation is also affected by other factors such as configurational structure, copolymer ratio, crystallinity, molecular weight, morphology, stresses, amount of residual monomer, porosity, and site of implantation [15].

Another problem that has been highlighted in the tissue engineering research field is the speed of vascularization into the engineered construct after its implantation. Most tissues in the body rely on blood vessels to supply the individual cells with nutrients and oxygen. For an in vivo tissue to grow beyond 100-200 $\mu \mathrm{m}$ (the maximum thickness for diffusion limit of oxygen), new blood-vessel formation is required, and this also holds for tissue-engineered structures. During in vitro culture, thicker tissueengineered structures can be supplied with nutrients, for instance in perfusion bioreactors. However, after their implantation in vivo, the supply of oxygen and nutrients to the implant is very often controlled by diffusion processes that can only supply cells in the proximity of the next capillary. For these reasons, the successful use of tissueengineered constructs is currently limited to thin or avascular tissues, such as skin or cartilage, for which postim- plantation neo-vascularization from the host are sufficient to meet the demand for oxygen and nutrients. To succeed in the application of tissue engineering for thicker tissues, such as bone and muscle, the problem of vascularization has to be solved [16].

Going to the protocols for the synthesis and preparation of graft to be used in tissue engineering applications, in the last decade phase separation techniques have been widely employed in order to obtain porous scaffold for tissue engineering application [17-20]. The advantage of these methods consists in the generation of a wide range of porosities, pore sizes, and morphologies, by fine tuning of the experimental parameters. In other words, it has been clearly shown how it is possible to control accurately the final morphology of the foams.

The morphological characteristics that the scaffold must fulfill in order to ensure a proper level of nutrient and metabolite exchange during blood flow still have to be thoroughly clarified. All things considered, it should be stressed that wall thickness, homogenous or increased porosity and pore size contribute to the final goal of achieving a structure guaranteeing simultaneously [21]: (i) cell spreading and population both in the scaffold walls (fibroblasts, smooth muscle cells, etc.) and on the internal surface of the artificial vessel [endothelial cells (EC)]; this implies that the porosity in the scaffold wall must be open, with an average porosity above $60 \%$ and average pore size around $10 \mu \mathrm{m}$ [22, 23]; (ii) a blood flow rate compatible with the physiology of the grafting site with the proper exchange of nutrients and metabolites; this condition can be satisfied only if the average pore size in the internal surface of the artificial blood vessels is below $1 \mu \mathrm{m}$, otherwise the cultured EC could migrate too early towards the internal layers of the vascular graft.

To comply with the aforementioned requirements, it is mandatory to deal with a preparation methodology allowing one to obtain a wide span of different morphologies (wall thickness, porosity, and pore size). Phase separation techniques seem to represent the best candidates for achieving all these objectives. Different typologies of phase separation are known such as thermally induced phase separation, air-casting of a polymer solution, precipitation from the vapor phase, and immersion precipitation $[24,25]$.

Examples of synthetic blood vessels prepared via different methods are shown in Refs. 26-29, as schematically reported in Table 1 , where four preparation protocols using a set of various materials are listed, together with the final wall thickness obtained.

In this article, porous and biodegradable PLLA scaffolds for vascular tissue engineering applications, with a vessel-like shape, were produced by a two-step experimental protocol, including a dip-coating of a viscous polymer solution around a nylon fiber and a diffusion induced phase separation (DIPS) by immersion into an antisolvent bath. The first step (dip-coating) allows one to generate the tubular structure of the vascular graft, 
whereas the second (DIPS) is responsible for the interesting level of porosity and for the surface microporosity as potentially ensuring nutrient and metabolites exchange during blood flow. The as-prepared scaffold can be utilized either as vascular grafts, or embedded into a porous scaffold for the regeneration of an injured tissue as a pseudo peripheral circulatory system, with the aim of promoting a rapid vascularization of the whole tissue engineered construct. This latter approach will be discussed elsewhere going beyond the scope of the present article, focusing on the former topic, i.e. the use of PLLA scaffolds as engineered vascular prostheses. With this respect, preliminary in vitro cell culture test showed a successful assembling of the EC, a first step toward the design and preparation of more complex vascular structures.

\section{EXPERIMENTAL}

\section{Scaffold Preparation and Characterization}

The PLLA vessel-like scaffolds (PLLA Resomer ${ }^{\mathrm{TM}}$, kindly supplied by Boehringer Ingelheim Pharma KG) were obtained by performing a DIPS process, after a dipcoating, around a nylon fiber with a diameter of $\sim 600$ $\mu \mathrm{m}$. In a first step (dip-coating), the fiber was first immersed into a PLLA/dioxane solution (dip-coating bath) at a constant temperature and was slowly pulled-out at a constant rate from the solution (Fig. 1). Via this procedure, the nylon fiber was coated by a layer of a viscous PLLA/dioxane binary solution. In a second stage (DIPS), the fiber was immersed into a second bath (DIPS or coagulation bath), containing pure water, or dioxane/water solutions at different ratios, at the same temperature, for a well-defined time interval (Fig. 2). This immersion into a phase separation bath is responsible for the porous structure of the tubular scaffold, owing to the diffusion of the nonsolvent (water) from the bath to the polymeric solution and of the solvent (dioxane) in the opposite sense. The microporous structure achieved is necessary for

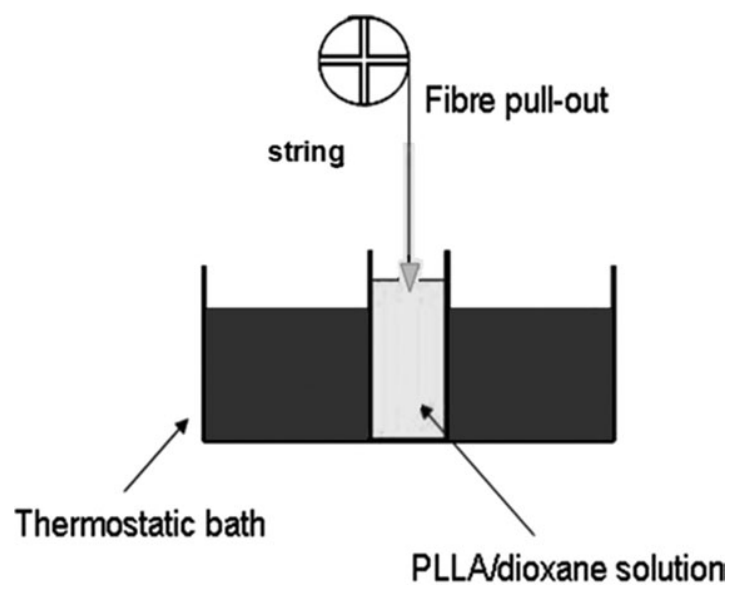

FIG. 1. Schematic representation of the dip-coating bath.

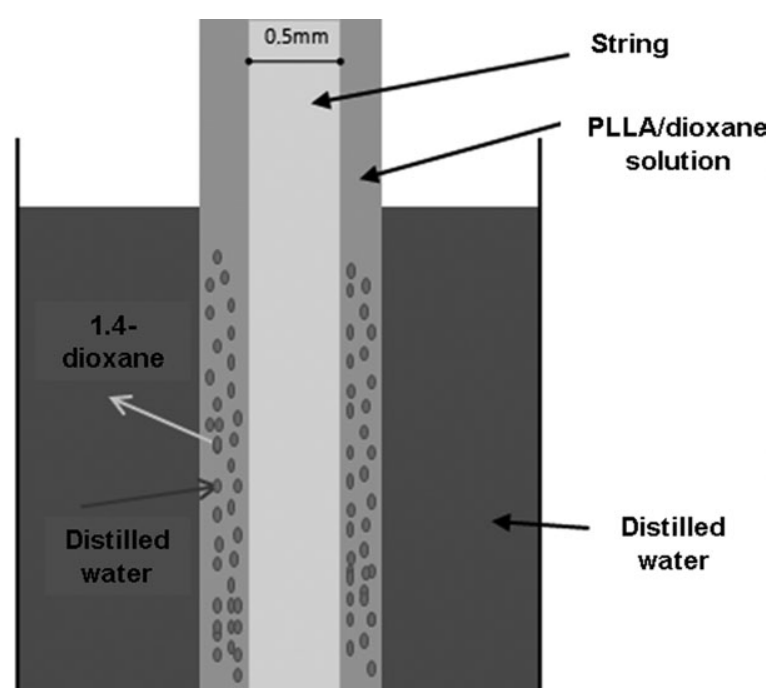

FIG. 2. Schematic representation of the diffusion induced phase separation (DIPS) bath.

nutrients and metabolites transport from the inner of the artificial vessel outwards and vice versa. Finally, the fiber was extracted from the bath, eventually rinsed in distilled water, and dried at $80^{\circ} \mathrm{C}$ for $48 \mathrm{~h}$.

The as obtained scaffolds were analyzed by scanning electron microscopy (SEM) with a Philips 505 Microscope on sample cross-section fractured in liquid nitrogen and gold stained.

An estimation of the wall thickness of the scaffold was carried out by an analysis of at least three SEM micrographs for each sample, in order to have a statistically representative sample.

\section{Cell Culture and Observation}

The continuous human endothelial cell line ECV304 (IZSBS BS CL 137), purchased from the "Istituto Zooprofilattico" of Brescia, Italy, was cultured in Medium 199 (GIBCO, Grand Island, NY) supplemented with 2 $\mathrm{m} M$ L-glutamine, 1 unit $/ \mathrm{ml}$ penicillin, $10 \mathrm{mg} / \mathrm{ml}$ streptomycin, 5 units/ml heparin, and $10 \%$ bovine serum (HyClone, Logan, UT).

ECV304 cell were seeded inside the PLLA scaffold pretreated with acidic $\left(0.02 \mathrm{~N} \mathrm{CH}_{3} \mathrm{COOH}\right)$ type-I collagen fibrils solution, buffered with complete media at $1.2 \times$ $10^{7}$ cell/ml $(200 \mu \mathrm{l})$. Media were exchanged every $2 / 3$ days.

Scaffolds were fixed for $10 \mathrm{~min}$ at room temperature in culture media buffer supplemented with $4 \%$ formaldehyde; washed three times in PBS containing $\mathrm{Ca}^{++}$and $\mathrm{Mg}^{++}$. Tubes were sectioned across the transverse direction in smaller pieces about 8-10 mm long, longitudinally opened and analyzed by different staining method. Some were directly stained with a solution of $0.2 \%$ Coomassie Blue R-250 in a ternary solution $\mathrm{CH}_{3} \mathrm{OH}: \mathrm{CH}_{3} \mathrm{COOH}: \mathrm{H}_{2} \mathrm{O}$ (5:1:5) for $3 \mathrm{~min}$ and washed in $\mathrm{H}_{2} \mathrm{O}$ (at least 5 times). 
Some other samples were incubated in a solution containing FITC-Phalloidin (0.006\%-Sigma) and ethidium bromide $\left(10 \mu \mathrm{g} / \mathrm{ml}-\right.$ Sigma) $2 \mathrm{hr}$ at $4^{\circ} \mathrm{C}$; thereafter washed 5 times in PBS containing $\mathrm{Ca}^{++}$and $\mathrm{Mg}^{++}$and mounted with mounting buffer (glycerol $80 \%$ plus $\mathrm{NaN}_{3} 0.02 \%$ in Tris buffer $0.5 M$ at $\mathrm{pH} 7.4$ ).

Prepared samples were observed under an Olympus Floview FV300 laser confocal microscope (2-mm laser sections).

For the SEM observation, the samples were fixed by following the same procedure as described above, rinsed in phosphate buffer saline (PBS) $1 \times$ and dehydrated in increasing concentrations of ethanol. Dehydrated samples were gold sputter coated and observed by using a PHILIPS 505 SEM.

\section{RESULTS}

Influence of Operating and Experimental Parameters on Scaffold Morphology

Polymer concentration, solution apparent viscosity, as well as fiber drawing speed and process temperature are expected to control scaffold's final wall thickness and the global morphology. The thickness of the scaffolds obtained through this methodology is reported in Table 2 for various experimental conditions. It is interesting to compare those data with the thickness of vascular scaffolds prepared via different techniques (electrospinning, TIPS, molding), as shown in Table 1. As it can be easily noticed, the protocol described in this article allows one to better tune scaffold wall thickness, covering a wider span of dimensions (from 10 to about $80 \mu \mathrm{m}$ ).

TABLE 2. Experimental condition for the preparation of the vessel like scaffold.

\begin{tabular}{|c|c|c|c|c|c|c|}
\hline $\begin{array}{l}\text { Sample } \\
\text { code }\end{array}$ & $\begin{array}{c}T \\
\left({ }^{\circ} \mathrm{C}\right)\end{array}$ & $\begin{array}{l}\text { DIPS } \\
\text { time } \\
(\mathrm{min})\end{array}$ & $\begin{array}{l}\text { PLLA conc. } \\
\text { (dip-coating } \\
\text { bath) wt } \%\end{array}$ & $\begin{array}{c}\text { Dioxane/ } \\
\text { water ratio } \\
\text { (DIPS bath) }\end{array}$ & $\begin{array}{l}\text { Pull out } \\
\text { velocity } \\
(\mathrm{cm} / \mathrm{min})\end{array}$ & $\begin{array}{c}\text { Thickness } \\
\qquad(\mu \mathrm{m})\end{array}$ \\
\hline FER1 & 60 & 10 & 8 & $0 / 100$ & 2.4 & 10 \\
\hline FER2 & 60 & 10 & 8 & $0 / 100$ & 4.8 & 20 \\
\hline FER3 & 60 & 10 & 8 & $0 / 100$ & 7.2 & 24 \\
\hline FER4 & 60 & 10 & 8 & $0 / 100$ & 14.4 & 43 \\
\hline FER5 & 60 & 10 & 8 & $0 / 100$ & 19.2 & 50 \\
\hline PC1 & 60 & 10 & 6 & $0 / 100$ & 7.2 & 3.25 \\
\hline PC2 & 60 & 10 & 6 & $0 / 100$ & 14.4 & 3.6 \\
\hline $\mathrm{T} 1$ & 75 & 10 & 8 & $0 / 100$ & 7.2 & 10 \\
\hline $\mathrm{T} 2$ & 45 & 10 & 8 & $0 / 100$ & 7.2 & 35 \\
\hline T3 & 30 & 10 & 8 & $0 / 100$ & 7.2 & 78 \\
\hline DWR1 & 60 & 10 & 8 & $10 / 90$ & 14.4 & \\
\hline DWR2 & 60 & 10 & 8 & $20 / 80$ & 14.4 & \\
\hline DWR3 & 60 & 10 & 8 & $50 / 50$ & 14.4 & \\
\hline DWR4 & 60 & 10 & 8 & $60 / 40$ & 14.4 & \\
\hline
\end{tabular}

The sample code indicates the experimental parameter varied (FER, fiber extraction rate; PC, polymer concentration; $T$, process temperature; DWR, dioxane/water ratio).
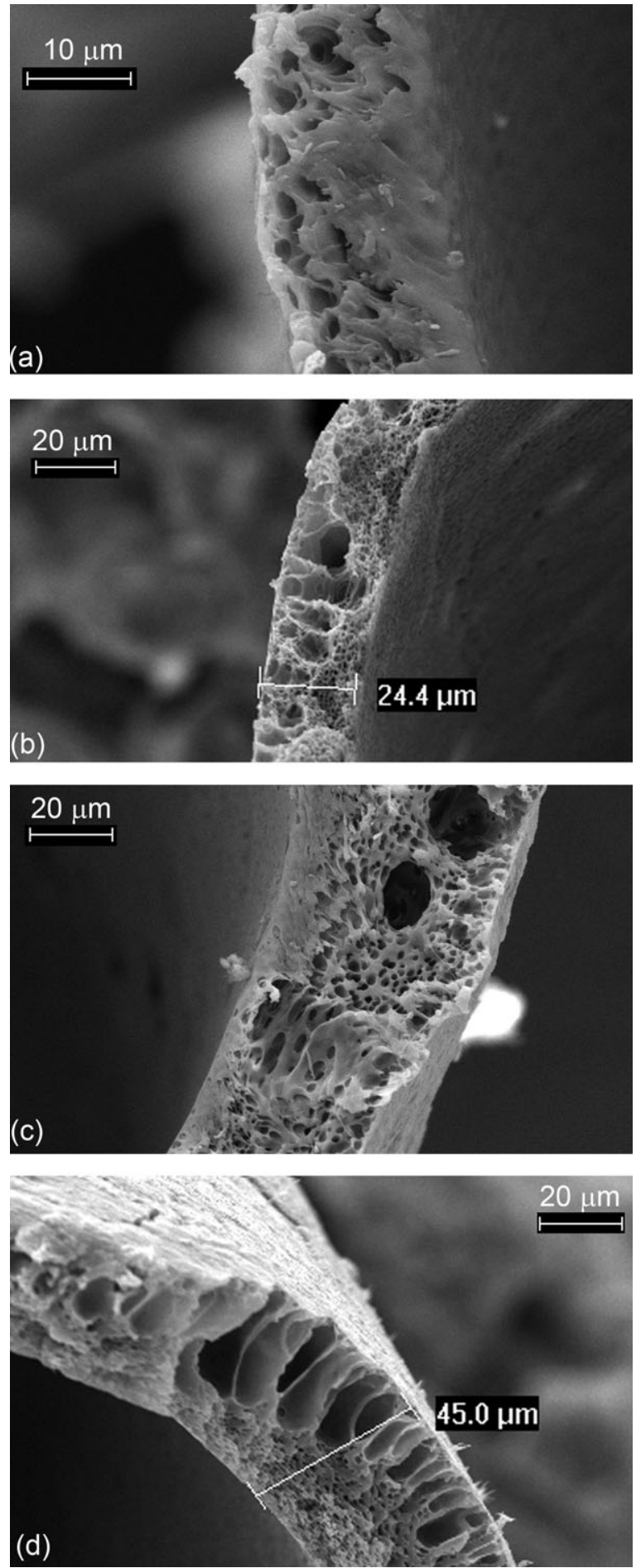

FIG. 3. SEM micrograph of a sample prepared at different fiber extraction rates: (a) $4.8 \mathrm{~cm} / \mathrm{min}$ (FER2), (b) $7.2 \mathrm{~cm} / \mathrm{min}$ (FER3), (c) $14.4 \mathrm{~cm} /$ min (FER4), and (d) $19.2 \mathrm{~cm} / \mathrm{min}$ (FER5).

Fiber Extraction Rate. An accurate analysis of the scaffold's wall structure via SEM revealed that the wall thickness is closely related to the speed at which the fiber is pulled out from the dip-coating bath (Fig. 3a-d).

Data shown in Table 2, which report the wall thickness measured by SEM images analysis at various extraction 


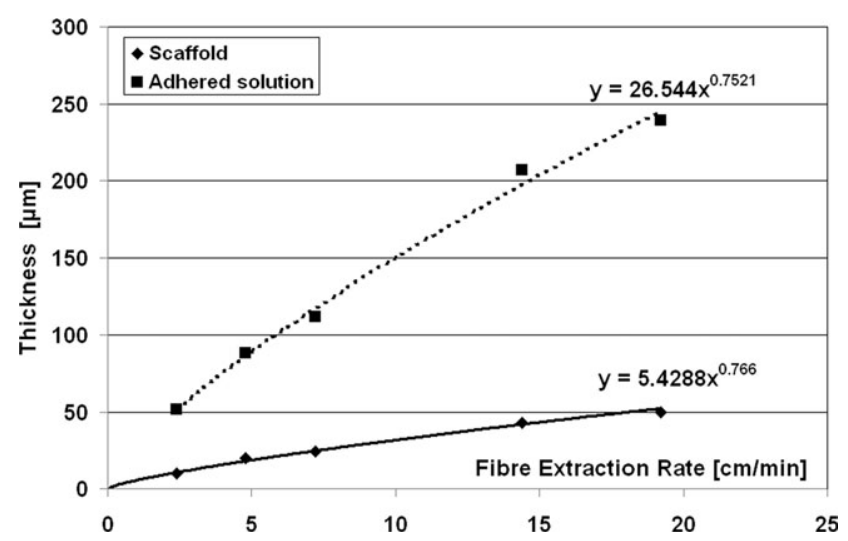

FIG. 4. Wall thickness and thickness of the extracted solution trends by varying fiber extraction rate at $60^{\circ} \mathrm{C}$ for $10 \mathrm{~min}$.

rates, indicate that wall thickness increases upon increasing the extraction velocity. This behavior can be essentially explained the increased thickness of the solution adhering to the fiber upon incrementing fiber extraction rate. It was chosen to operate within a range of extraction rates from 2.4 to $19.2 \mathrm{~cm} / \mathrm{min}$. As a matter of fact, rates slower than $2.4 \mathrm{~cm} / \mathrm{min}$ could lead to significant solvent evaporation during the dip-coating process due to the significant exposure time to atmosphere. Conversely, all the scaffolds produced with extraction rates faster than 19.2 $\mathrm{cm} / \mathrm{min}$ did not result homogeneous, mainly because of fluid-dynamic instabilities.

Table 2 reports the experimental conditions employed to prepare the scaffolds.

The correlation between wall thickness and fiber extraction velocity is reported in Fig. 4 (continuous line). By operating via this discontinuous technique, the portion of the fiber extracted first from the dip-coating solution bath remains exposed to the atmosphere longer with respect to the region extracted at the end. This exposition could lead to a significant evaporation of the dioxane adhered to the fiber, thus creating a different final morphology between the beginning and the end of the graft.

To verify if this phenomenon could really affect the final characteristics of the scaffold, short samples $(1 \mathrm{~cm})$, at the lowest extraction rate (longest residence time in the air), were prepared. Moreover, after extraction, instead of being immediately immersed in nonsolvent, the wet fiber was exposed to atmosphere for different time intervals (30 sec, $1 \mathrm{~min}, 2 \mathrm{~min}$, etc.) and subsequently immersed in the nonsolvent. The SEM analysis of the scaffold obtained via these tests showed that the morphology of the scaffold did not undergo significant changes, which confirms a negligible solvent evaporation from the fiber surface during the dip-coating process (data not shown).

PLLA Concentration. By changing the PLLA concentration in the dip-coating bath, large differences among the various samples were found.
As a matter of fact, a decrease in the PLLA concentration in the dip-coating solution determines both a lower solution viscosity and a reduced amount of polymer surrounding the nylon fiber. Those two effects significantly influence the final thickness of the wall of the scaffold. Samples were not easy-to handle when using a 4\% PLLA solution, due to the very thin scaffold wall. As expected, the samples obtained using a 6\% PLLA solution presented a lower thickness (few microns) with respect to the samples prepared under the same conditions by using a $8 \%$ (wt/wt) PLLA/dioxane solution (Fig. 5 and Table 2).

Processing Temperature. Also processing temperature plays a crucial role. Figure 6 clearly shows that, the lower the temperature, the higher the thickness of the scaffold. As viscosity is strongly dependent on temperature. When decreasing the temperature, solution viscosity increases determining, in its turn, an increment of the adhered solution.

Composition of the DIPS Bath. The last experimental parameter investigated was the composition of DIPS bath. In Fig. 7, SEM micrographs of samples prepared by changing the water/dioxane ratio of the DIPS bath are shown. It is easy to notice that the wall thickness of the various samples remains almost constant while the average diameter of the pores across the thickness increases, when raising the dioxane to water ratio.

It can be postulated that the presence of dioxane in the second bath slows down the solvent/nonsolvent exchange, with a decrease in terms of the global process rate. In
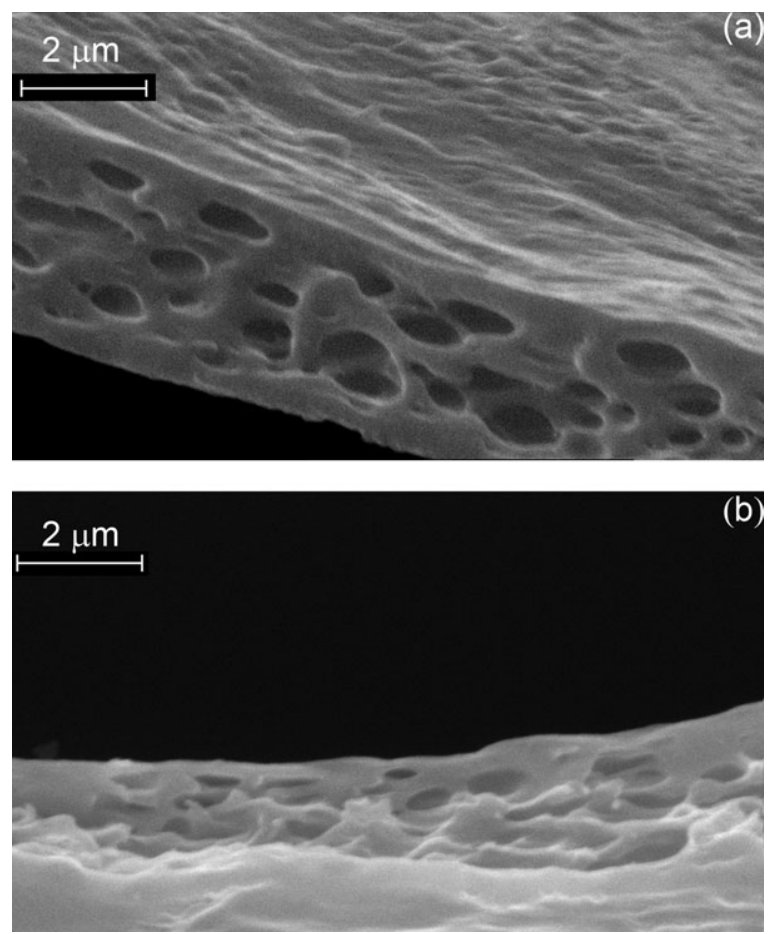

FIG. 5. Vessel-like scaffolds prepared with a $6 \%$ PLLA/dioxane solution, $T=60^{\circ} \mathrm{C}, t=10 \mathrm{~min}$, at different fiber extraction rates: (a) 7.2 $\mathrm{cm} / \mathrm{min}(\mathrm{PC} 1)$ and (b) $14.4 \mathrm{~cm} / \mathrm{min}(\mathrm{PC} 2)$. 

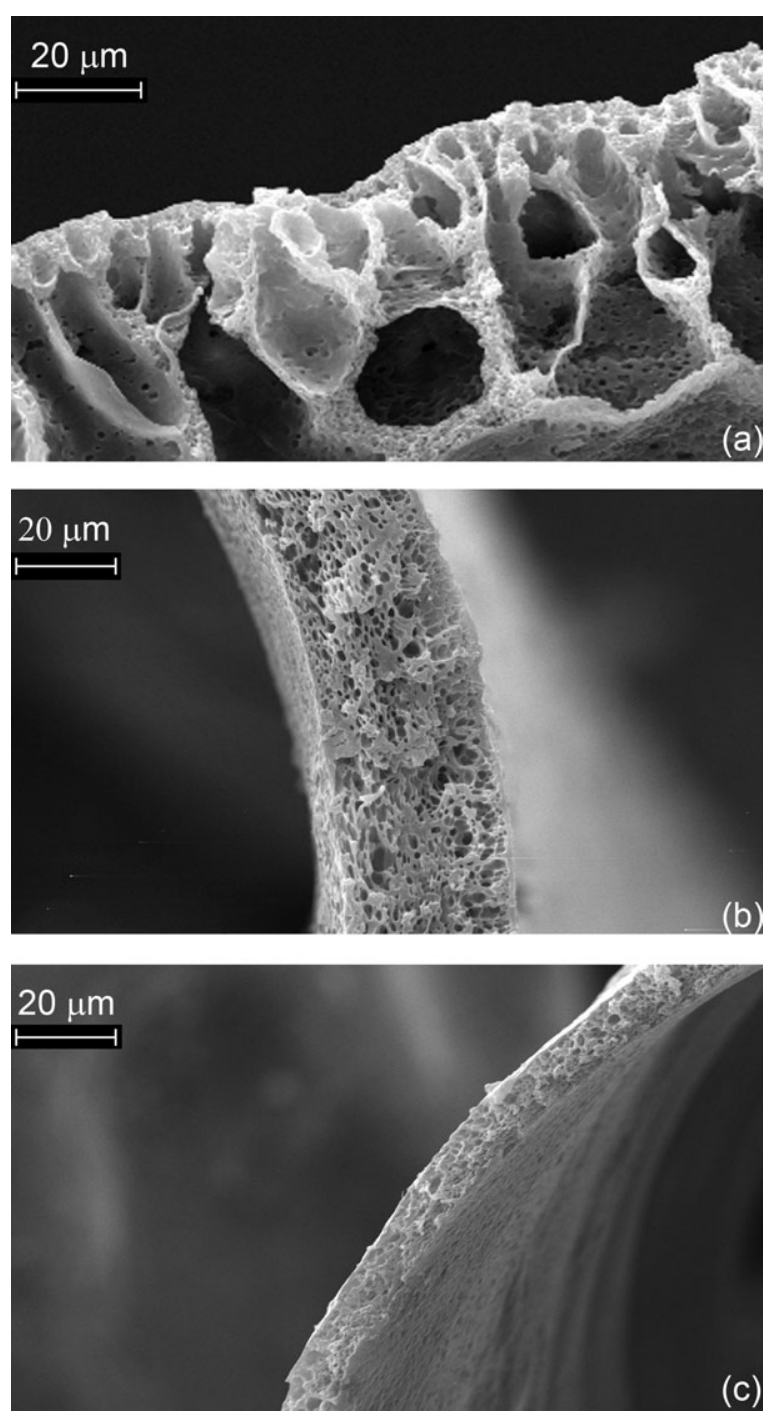

FIG. 6. Vessel-like scaffolds prepared with a $8 \%$ PLLA/dioxane solution pulled out at $7.2 \mathrm{~cm} / \mathrm{min}$ at different temperatures: (a) $75^{\circ} \mathrm{C}$ (T1) (b) $45^{\circ} \mathrm{C}$, (T2), and (c) $30^{\circ} \mathrm{C}$ (T3).

those conditions, the system spends more time in the growth and nucleation stage, leading to larger pores with respect to the pure water bath, thus leading to larger pores with respect to the pure water bath.

To sum up, the data presented indicate that all the parameters investigated influence the final morphology of the scaffolds. On the one hand, by changing the fiber extraction rate during the dip-coating stage, the PLLA/ dioxane ratio in the dip-coating bath and the temperature of the process is possible to accurately control the wallthickness; on the other hand, the average pore dimension can be properly tuned by varying the dioxane to water ratio of the DIPS bath.

\section{Thickness of Dip-Coating Solution vs. Scaffold Thickness}

Thickness of Adhered Solution. By measuring the weight of the samples, it was possible to estimate the thickness of the solution adhering to the fiber surface during the dip-coating process. The use of this technique is justified by the difficulty to measure the thickness of the solution immediately after the dip-coating, as this is a thin film of polymer solution, not allowing easy optical determinations. Moreover, at the highest temperatures, the
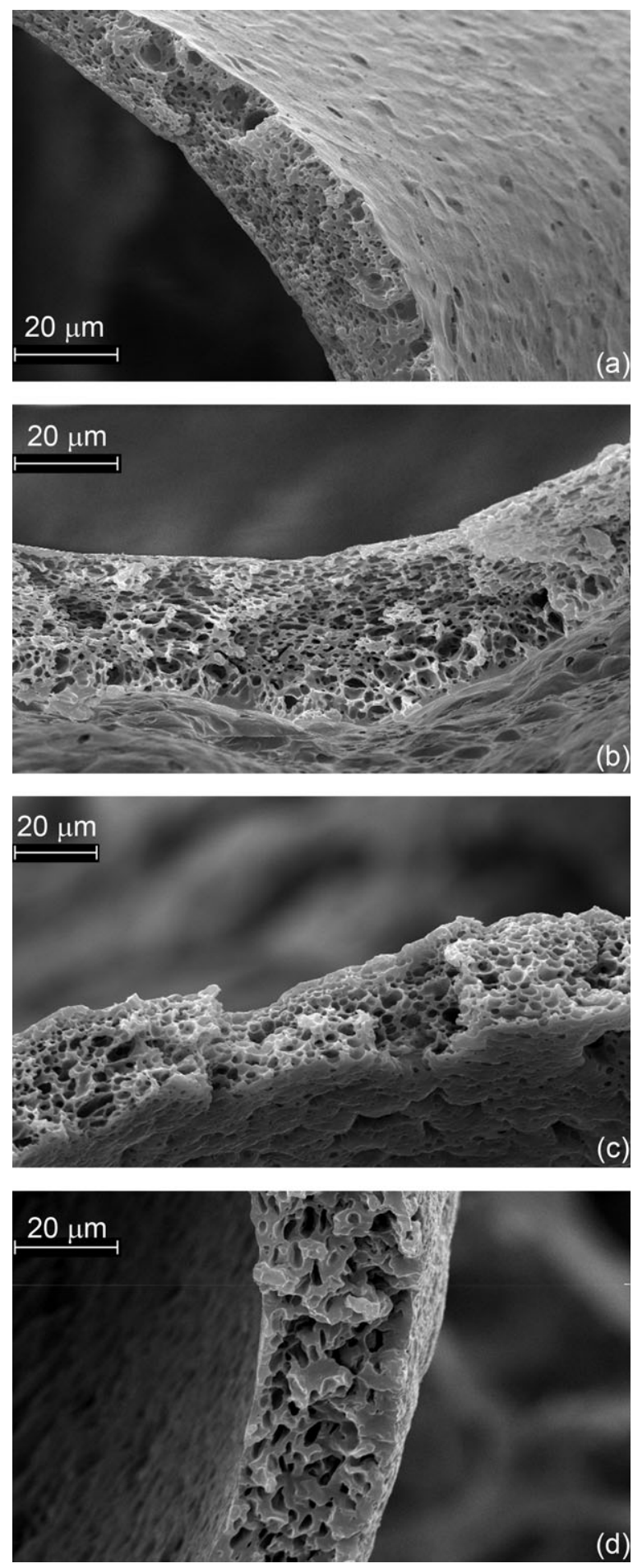

FIG. 7. Vessel-like scaffolds prepared with a 8\% PLLA/dioxane solution, pulled out at $14.4 \mathrm{~cm} / \mathrm{min}$ at $60^{\circ} \mathrm{C}$ with different dioxane/water ratios in DIPS bath: (a) 10/90 (DWR1), (b) 20/80 (DWR2), (c) 50/50 (DWR3), and (d) 60/40 (DWR4). 
solvent in the solution (dioxane) tends to evaporate and thereby the thickness of the wall reduces, making even more difficult (and less reliable) optical measurements. It is therefore more recommended to operate on the final sample, the solid polymer scaffold, attaining the volume per unit length through the following relationship (assuming a negligible amount of PLLA lost during the DIPS process):

$$
\frac{V}{L}=\frac{\text { weight of PLLA per unit length }}{\rho_{\text {sol }} \times \text { PLLA weight fraction }}
$$

The density of the solution was calculated as follows:

$$
\rho_{\text {sol }}=\frac{1}{\frac{1}{\rho_{\text {diox }}} \omega_{\text {diox }}+\frac{1}{\rho_{\text {PLLA }}} \omega_{\text {PLLA }}}
$$

where $\rho_{\text {sol }}$ is the density of the solution, $\rho_{\text {diox }}$, and $\omega_{\text {diox }}$, are the density and the weight fraction of dioxane, respectively and $\rho_{\text {PLLA }}$ and $\omega_{\text {PLLA }}$, are the density and the weight fraction of PLLA, respectively.

Finally, knowing the radius of the fiber, it was possible to calculate the thickness of the extracted solution based on simple geometrical considerations:

$$
\delta=\sqrt{r^{2}+\frac{1}{\pi} \frac{V}{L}}-r
$$

where $\delta$ is the thickness of the solution extracted, $r$ is the radius of the nylon fiber, and $\frac{V}{L}$ is the volume of extracted solution per unit length.

The thicknesses of the adhered solution extracted are summarized in Fig. 4 (dotted line).

Those data, compared with the thickness of the scaffold measured via SEM, allow one to estimate the decrease of the thickness due to the DIPS process.

When comparing the data reported in Fig. 4, it is evident that the thickness of the adhered solution and the final scaffold's thickness follow the same trend upon increment the fiber extraction rate, with a power low dependence exhibiting an exponent very close to 3/4 in both cases.

On the basis of those data, it was possible to compute the ratio between the scaffold's thickness and the thickness of the adhered solution for each rate explored, which resulted constant, regardless the extraction rate. In other words, whatever the initial thickness of the solution, during the DIPS process, the thickness reduces always by the same fraction and therefore the resulting porosity remains constant, as outlined in the following paragraph.

Scaffold's Porosity. The thickness of the scaffold and its weight allow one to calculate the degree of porosity $(P)$ of the scaffolds, defined as:

$$
P=\frac{V_{v}}{V_{\text {tot }}}
$$

where $V_{\mathrm{v}}$ (the volume of voids) is given by:

$$
V_{v}=V_{\text {tot }}-V_{\text {PLLA }}
$$

The degree of porosity can be written also as:

$$
P=\frac{V_{\text {tot }}-V_{\text {PLLA }}}{V_{\text {tot }}}=1-\frac{V_{\text {PLLA }}}{V_{\text {tot }}}
$$

Both the volumes can be defined per unit length $L$ :

$$
\text { porosity }=1-\frac{V_{\text {PLLA }} / L}{V_{\text {tot }} / L}
$$

The volume of PLLA can also be rewritten as:

$$
V_{\text {PLLA }}=\frac{W_{\text {PLLA }}}{\rho_{\text {PLLA }}}
$$

While the volume of the scaffold per unit length, based on geometric considerations, is:

$$
\frac{V}{L}=\pi *\left(\delta^{2}+2 r \delta\right)
$$

Upon making the necessary substitutions one gets:

$$
P=1-\frac{\frac{W_{\mathrm{PLLA}}}{L} / \rho_{\mathrm{PLLA}}}{\pi *\left(\delta^{2}+2 r \delta\right)}=1-\frac{\frac{W_{\mathrm{PLLA}}}{L}}{\rho_{\mathrm{PLLA}} * \pi *\left(\delta^{2}+2 r \delta\right)}
$$

The porosity maintains almost constant for all the different conditions, with values ranging from 62 to $68 \%$. This is an appreciable result when combined with the low thickness of the scaffold (below $50 \mu \mathrm{m}$ ) and a sufficient mechanical strength, which allows the handling of the artificial vessel. Moreover, the results of porosity and pores size are in line with the expectations discussed in the introduction paragraph, as for cell spreading and population both in the scaffold walls (fibroblasts, smooth muscle cells, etc.) and in the internal surface of the artificial vessel (EC), and the possibility to apply a blood flow rate compatible with the physiology of the grafting site ensuring the proper exchange of nutrients.

The degree of porosity was also calculated for the scaffolds prepared by varying the dioxane/water ratio in DIPS bath. As shown in Fig. 8, the porosity increases upon raising the amount of dioxane in DIPS bath [24, 25].

\section{ECV Culture}

A cell culture inside the vessel-like scaffolds was carried out, by using EC, which are the solely components of capillary bed and the first to form during embryonic development. Figure 9 illustrates a laser confocal microscope image of a longitudinal section of a scaffold with 


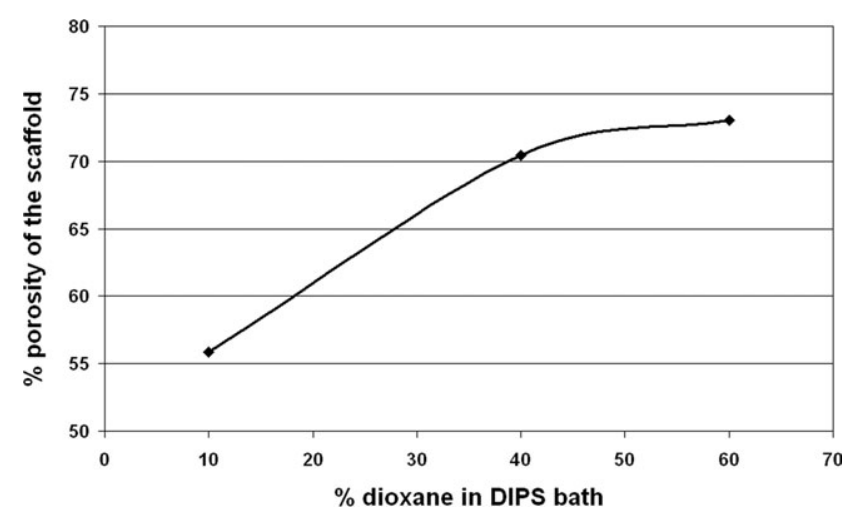

FIG. 8. Degree of porosity as a function of dioxane/water ratio of the DIPS bath.

EC grown for 3 weeks. The circles are the nuclei of the cells, while the other bright areas indicate the actin, a cytoskeleton protein. The image clearly shows that after 21 days the internal lumen of the scaffold is totally covered by EC, which have organized themselves into a well differentiate vessel structure. Cells shown to form stable cell-cell interactions and spindle membrane protrusion, characteristic of mesenchymal endothelial phenotype, were not observed. This result was confirmed by the ESM analysis of the same section (data not shown).

Figure 10 shows the same scaffold stained with blue coomassie and observed under an optical microscope. Also in these pictures the layer of EC appears well defined.

These experiments suggest that the scaffold produced could be usefully employed in vascular tissue engineering applications.

\section{Modeling}

To assess the dependence of film thickness on fiber extraction rate as well as on the relevant parameters, the fluid-dynamics of the process was modeled.

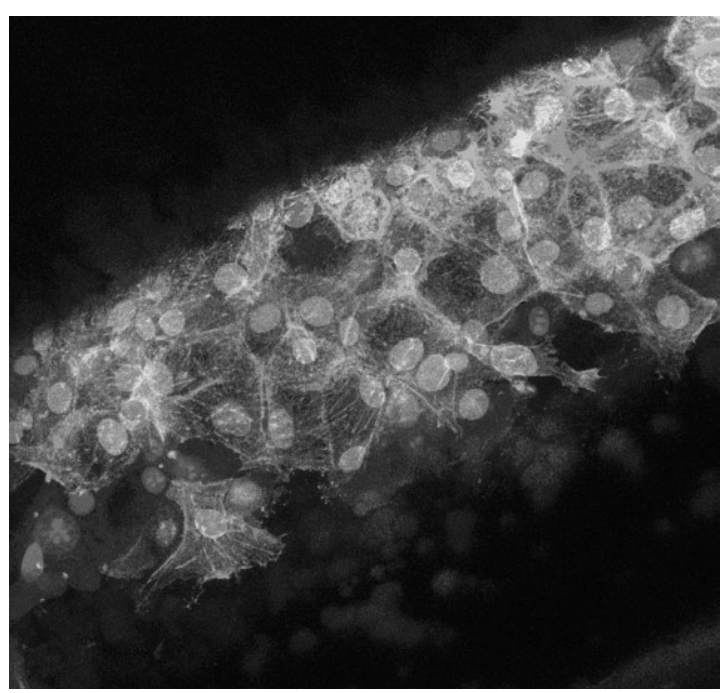

FIG. 9. Confocal microscope image of the EC grown inside the scaffold for 21 days.

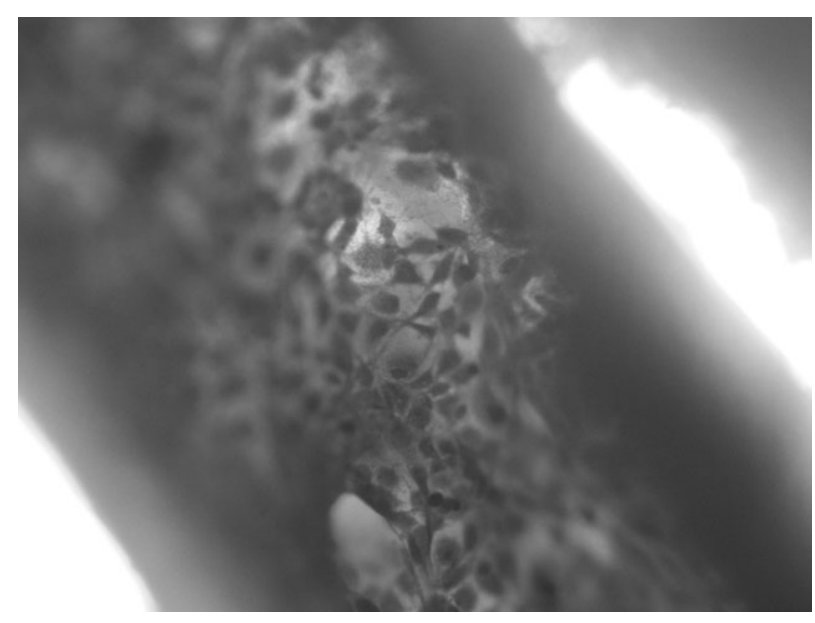

FIG. 10. Toluidine blue staining of the ECV grown inside the scaffold for 21 days.

The results of modeling were compared with experimental data and discussed.

The starting point was the use of a simplified model based on reasonable approximations; the advantage of this approach is the possibility of obtaining analytical solutions valid also to test numerical solutions, needed when more complex modeling is applied.

The hypotheses and simplifications were the following: incompressible Newtonian fluid, flat geometry (polymer solution film thickness $\delta$ small with respect to the fiber radius $R$ ), negligible surface tension effects, lubrication approximation (viscous stresses predominating) and steady state conditions.

The flat geometry of the system can be schematized as shown in Fig. 11.

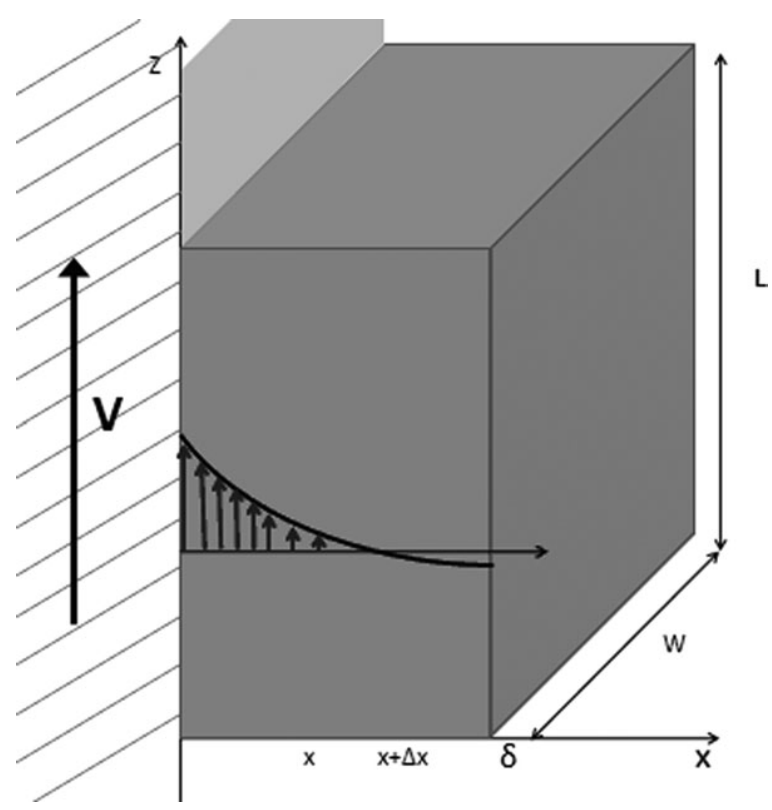

FIG. 11. Schematic representation of the flat geometry assumed for the system. 
Under the preceding limitations, the projection of the momentum balance equation, for viscous Newtonian fluid [30] along the $z$-axis (velocity direction) reduces to:

$$
\frac{\partial^{2} v_{z}}{\partial x^{2}}=\frac{\rho g}{\mu} ; \quad \text { B.C. }\left\{\begin{array}{l}
x=0 \Rightarrow v_{z}=V \\
x=\delta \Rightarrow \frac{\partial v_{z}}{\partial x}=0
\end{array}\right.
$$

with the well known solution (Flow of a falling film [31]):

$$
v_{z}=V+\frac{\rho g \delta^{2}}{2 \mu}\left[\left(\frac{x}{\delta}\right)^{2}-2\left(\frac{x}{\delta}\right)\right] \text { with } \delta=\delta(z)
$$

On the other hand, the equation of continuity for an incompressible fluid provides:

$$
\frac{\partial v_{x}}{\partial x}+\frac{\partial v_{z}}{\partial z}=0
$$

Furthermore, the following restrictions on $v_{\mathrm{x}}$ apply:

$$
\left.v_{x}\right|_{x=0}=0 ; \quad \frac{\left.v_{x}\right|_{x=\delta}}{\left.v_{z}\right|_{x=\delta}}=\frac{d \delta}{d z}
$$

Integration of the Eq. 13 along the $x$ direction leads to:

$$
\begin{aligned}
& \int_{0}^{\delta} \frac{\partial v_{x}}{\partial x} d x+\int_{0}^{\delta} \frac{\partial v_{z}}{\partial z} d x=0 \\
& \left.v_{x}\right|_{x=\delta}-\left.v_{x}\right|_{x=0}+\int_{0}^{\delta} \frac{\partial v_{z}}{\partial z}=0
\end{aligned}
$$

and, replacing Eqs. 12 and 14 into Eq. 15, the following equation can be drawn:

$$
\frac{d \delta}{d z}\left(\left.v_{z}\right|_{x=\delta}\right)-\int_{0}^{\delta}\left(\frac{\rho g}{2 \mu} 2 x\right) \frac{d \delta}{d z} d x=0
$$

that can be now further substituted in $E q .12$ and integrated:

$$
\frac{d \delta}{d z}\left(V-\frac{\rho g \delta^{2}}{2 \mu}\right)-\frac{d \delta}{d z} \frac{\rho g}{2 \mu} \delta^{2}=0
$$

At the end, after easy simplifications, the relationship between the film thickness and the fiber extraction rate on the dependence on the other parameters, can be found as follows:

$$
V-\frac{\rho g}{\mu} \delta^{2}=0 ; \quad \delta=\sqrt{\frac{\mu V}{\rho g}}
$$

It is worth noticing that the film thickness $(\delta)$ depends on the square root of fiber extraction rate $(V)$.

The flow curve of the solution at $60^{\circ} \mathrm{C}$ was determined and a reasonable viscosity of the value of about $7 \mathrm{~Pa} \mathrm{~s}$

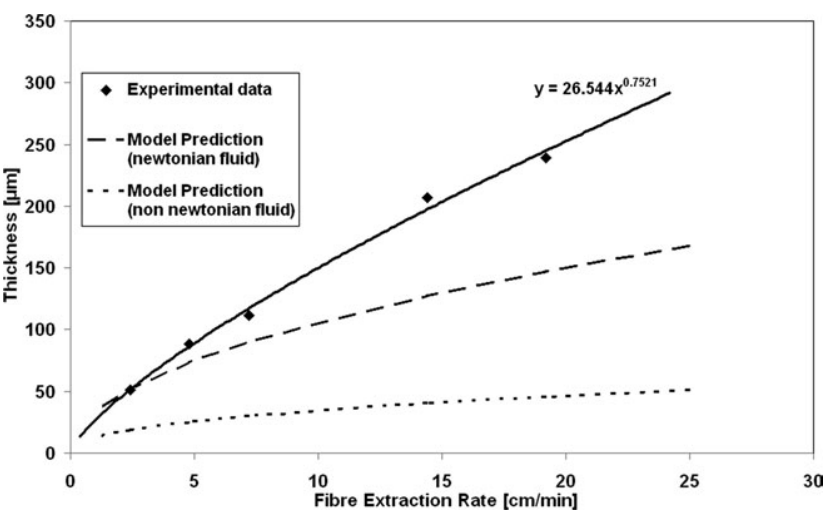

FIG. 12. Comparison between predicted and experimental data of polymer solution thickness for Newtonian and power law fluids.

was found at a shear rate of $5 \mathrm{~s}^{-1}$ (the average value), furthermore a density value of $1050 \mathrm{~kg} \mathrm{~m}^{-3}$ can be easy estimated for an ideal polymer solution.

As fiber extraction rate is known, since it was imposed and measured during the experiments, the values of the film thickness can be predicted. The comparison between data and predictions is reported in Fig. 12 (heavy dashed line), where a very good agreement is obtained at very low extraction rates; at higher values however, thickness is clearly underestimated by the model. Data show a dependence on extraction rate raised to the power 0.75 , which is larger than the value provided by the model.

The possibility that the approximations made will be the cause of the unsatisfactory agreement at high extraction rates was examined by adapting a more rigorous modeling.

The first simplification removed was the hypothesis of Newtonian fluid. A simple power law was then applied, by starting from the shear stress based formulation of the momentum balance equation [31]:

$$
\frac{\partial \tau_{x z}}{\partial x}=-\rho g ; \quad \text { B.C. } x=\delta \Rightarrow \tau_{x z}=0
$$

the solution being:

$$
\tau_{x z}=\rho g(\delta-x)
$$

the constitutive equation now will become:

$$
\tau_{x z}=-K\left(\frac{\partial v_{z}}{\partial x}\right)\left|\frac{\partial v_{z}}{\partial x}\right|^{n-1}
$$

considered that the shear stress is everywhere positive and the velocity gradient is everywhere negative, the sign can be transported inside the brackets:

$$
\tau_{x z}=K\left(-\frac{\partial v_{z}}{\partial x}\right)^{n}
$$




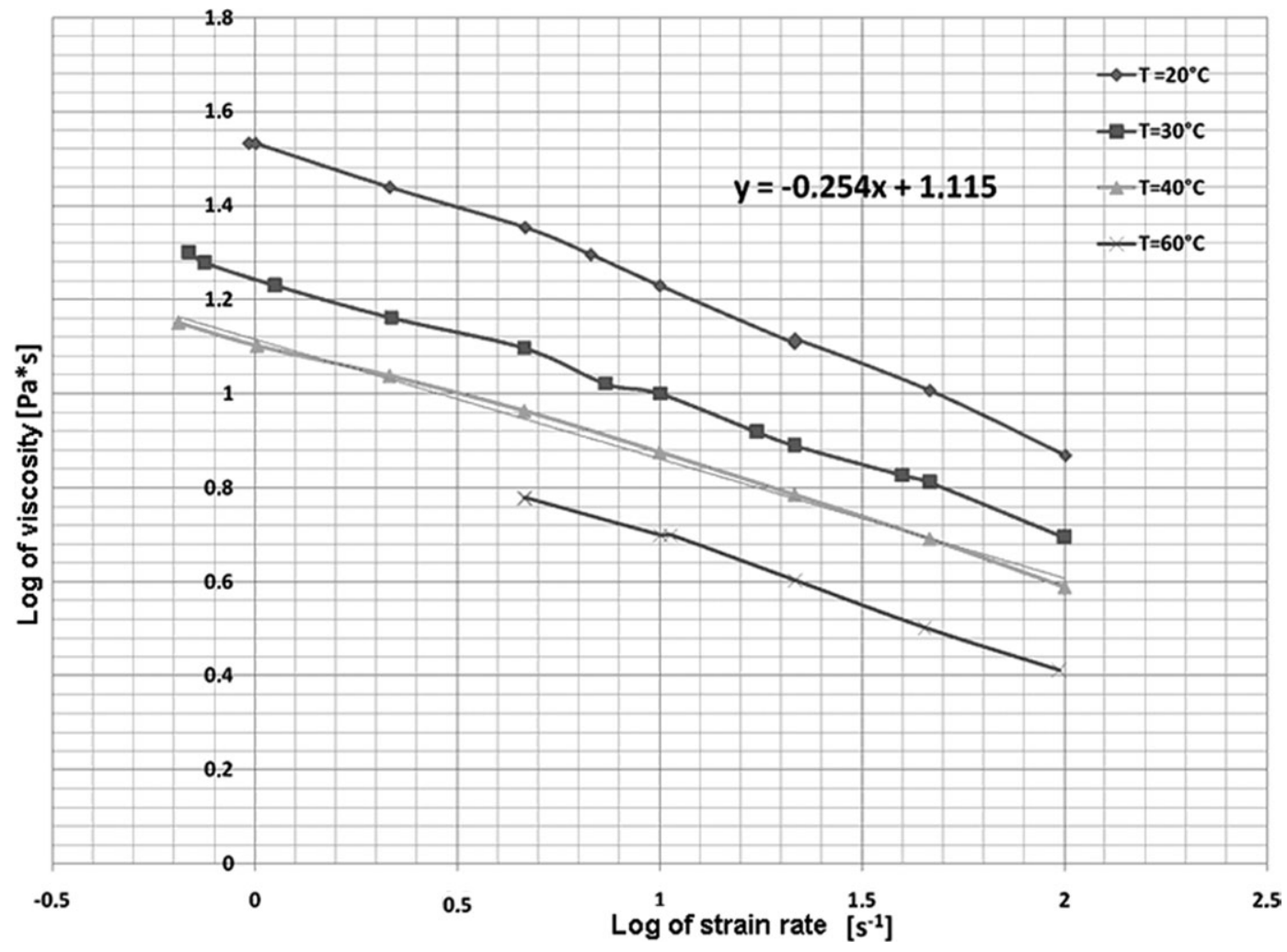

FIG. 13. Flow curve of the polymeric solution at various temperatures.

while the values of the constants $K$ and $n$ can be drawn from experimental data considered that, in a log-log plot, the power law is a straight line and a linear regression analysis can be applied:

$$
\eta=\frac{\tau_{x z}}{\frac{\partial v_{z}}{\partial x}}=\frac{\tau_{x z}}{\dot{\gamma}}=K \dot{\gamma}^{(n-1)}
$$

Figure 13 reports rheological data obtained in a stress rheometer (SR5 Rheometric Scientific) operating in a plate-plate geometry; applying a linear regression analysis and the following values were found: $n=0.746$ and $K=$ $1.115 \mathrm{~Pa} \mathrm{~s}^{0.746}$.

Upon replacing Eq. 22 in Eq. 20, the following differential equation is obtained:

$$
\rho g(\delta-x)=K\left(-\frac{\partial v_{z}}{\partial x}\right)^{n} \quad \text { B.C. } x=0 \Rightarrow v_{z}=V
$$

After variable separation, integration, and B.C. imposition, the equation leads to the following velocity distribution:

$$
v_{z}=V-\left(\frac{\rho g}{K}\right)^{\frac{1}{n}} \frac{n}{n+1} \delta^{\frac{n+1}{n}}\left[1-\left(1-\frac{x}{\delta}\right)^{\frac{n+1}{n}}\right]
$$

Applying this velocity distribution to continuity equation, under the same restrictions used before, after few steps the relationship between extraction rate and thickness can be found:

$$
V=\left(\frac{\rho g}{K}\right)^{\frac{1}{n}} \delta^{\frac{n+1}{n}}
$$

or

$$
\delta=V^{\frac{n}{n+1}}\left(\frac{K}{\rho g}\right)^{\frac{1}{n+1}}
$$

the solution for a Newtonian fluid being the special case with $n=1$.

Also in this case a comparison between data and predictions was carried out and is reported in Fig. 12 (thin dotted line) where, however, a bad agreement is obtained at all the extraction rate values examined; even worse than the pure Newtonian model. Moreover, the dependence of the thickness on extraction rate have, for pseudoplastic fluids like polymers, $(n<1)$ a power less than 0.5 .

As a further refining of the model, the cylindrical geometry was correctly accounted, essentially because the largest deviations of the "flat" model take place at large thickness, i.e., at large deviations from the flat geometry approximation. An analogs analytical solution, not reported here for sake of simplicity, can be obtained after a more complex derivation. However, the predictions leads to results worse than the flat model for Newtonian fluids. 
A straightforward way to show that this modeling choice cannot improve the predictions, can be easy derived by a simplification of the macroscopic momentum balance in a cylindrical geometry:

$$
\left.2 \pi R L \tau_{r z}\right|_{r=R}=-\left[(R+\delta)^{2}-R^{2}\right] L \rho g \pi
$$

where $R$ is the fiber radius and $L$ the length of fiber to which the macroscopic balance is applied. The dependence of shear stress on velocity gradient, for a Newtonian fluid, was in this case approximated by taking the average velocity gradient across the polymer solution thickness in spite of the fiber surface one.

$$
\left.\tau_{r z}\right|_{r=R}=-\left.\mu\left(\frac{\partial v_{z}}{\partial r}\right)\right|_{r=R} \approx-\mu \frac{V}{\delta}
$$

By introducing Eq. 29 in Eq. 28 the following relationship is obtained:

$$
\mu \frac{V}{\delta} 2 R \approx \rho g\left[(R+\delta)^{2}-R^{2}\right]
$$

That can be easy rearranged in:

$$
\delta^{2}\left(\frac{\delta}{2 R}+1\right) \approx\left(\frac{\mu V}{\rho g}\right)
$$

Although this relationship is only approximate, the trend found can be informative. It is worth noticing that, in Eq. 31, the thickness dependence on the extraction rate exhibits a power exponent ranging between $1 / 3$ and $1 / 2$, depending on the fiber radius. Then, also in this case, the predicted trend, is diverging from experimental data.

Concluding, the fluid-dynamics modeling of the dipcoating process appear to be shifty. Also the recent scientific literature [30] show that reliable solutions, either analytical and/or numerical, to the problem were not found despite the complexity of the modeling applied (elastoviscous rheology, interfacial tension effects etc.). In general all the predictions display a more or less pronounced underestimate of experimental data, thus indicating that all the models neglect some important but evasive influence.

\section{CONCLUSIONS}

An innovative method to synthesize porous biodegradable polymeric scaffolds for vascular tissue engineering applications via dip-coating of a nylon fiber with a PLLA/dioxane solution followed by DIPS was designed, set up and carried out. The as-prepared tubular scaffolds present a lumen of about 600 micron, a porous internal surface, and an open porosity across the wall thickness.

An accurate analysis of the preparation process revealed that it is possible to tune up the morphology of the scaffold (wall thickness, porosity, and average pore dimension), simply by varying some experimental parameters.

EC cultures were carried out in the scaffolds. The results demonstrated that the cells are able to grow inside the scaffold and after 21 days a vessel-like structure was generated, thus showing the potential of this method towards successful use in vascular tissue engineering applications.

Modeling predictions, either for Newtonian or powerlaw fluid, always displays a more or less pronounced underestimate of experimental data, with a predicted dependence of wall thickness on extraction rate of 0.5 , whereas experiments show a dependence on an exponent equal to 0.75 . The reasons for this discrepancy are under study and will be discussed separately.

\section{REFERENCES}

1. J.V. Tu, N. Engl. J. Med., 337, 1008 (1997).

2. M.S. Conte, FASEB J., 12, 43 (1998).

3. B.C. Isenberg, C. Williams, and R.T. Tranquillo, Circ. Res., 98, 25 (2006).

4. R.H. Schmedlen, W.M. Elbjeirami, A.S. Gobin, and J.L. West, Tissue Engineering, J.P. Fisher, A.G. Mikos, and J.D, Bronzino, Eds., CRC Press, Boca Raton, 26.1 (2007).

5. S. Venkatraman, F. Boey, and L.L. Lao, Prog. Polym., 33, 853 (2008).

6. D.N. Jones, R.B. Rutherford, T. Ikezawa, N. Nishikimi, H. Ishibashi, and T.A. Whitehill, J. Vasc. Surg., 14, 441 (1991).

7. C.O. Esquivel and F.W. Blaisdell, J. Surg. Res., 41, 1 (1986).

8. H.P. Greisler, D.U. Kim, J.B. Price, and A.B. Voorhees, Arch. Surg., 120, 315 (1985).

9. L.E. Niklason, J. Gao, W.M. Abbott, K.K. Hirschi, S. Houser, R. Marini, and R. Langer, Science, 284, 489 (1999).

10. O.E. Teebken, A.M. Pichlmaier, and A. Haverich, Eur. J. Vasc. Endovasc. Surg., 22, 139 (2001).

11. M. Cikirikcioglu, G. Bowlin, Y.B. Cikirikcioglu, K. Djebaili, T. Tatar, A. Kalangos, and B.H. Walpoth, Br. J. Surg., 92, 915 (2005).

12. M.J. Smith, M.J. McClure, S.A. Sell, C.P. Barnes, B.H. Walpoth, D.G. Simpson, and G.L. Bowlin, Acta Biomater., 4, 58 (2008).

13. E. Pektok, B. Nottelet, J.C. Tille, R. Gurny, A. Kalangos, M. Moeller, and B.H. Walpoth, Circulation, 118, 2563 (2008).

14. B. van der Lei, C.R. Wildevuur, F. Dijk, E.H. Blaauw, I. Molenaar, and P. Nieuwenhuis, J. Thorac. Cardiovasc. Surg., 93, 695 (1987).

15. H.Y. Cheung, K.T. Lau, T.P. Lu, and D. Hui, Compos. B, 38, 291 (2007).

16. J. Rouwkema, N.C. Rivron, and C.A. van Blitterswijk, Trends Biotech., 26, 434 (2008).

17. Y.S. Nam and T.G. Park, J. Biomed. Mater. Res., 47, 8 (1999) 
18. S. Li, V. La Carrubba, S. Piccarolo, D. Sannino, and V. Brucato, Polym. Int., 53, 2079 (2004).

19. S.Y. Kim, T. Kanamori, Y. Noumi, O.C. Wang, and T. Shinbo, J. Appl. Polym. Sci., 92, 2082 (2004).

20. F.C. Pavia, V. La Carrubba, S. Piccarolo, and V. Brucato, J. Biomed. Mater. Res. A, 86, 459 (2008).

21. F. Couet, N. Rajan, and D. Mantovani, Macromol. Biosci., 7, 701 (2007).

22. F.J. Hua, T.G. Park, and D.S. Lee, Polymer, 44, 1911 (2003).

23. T.M. Freyman, I.V. Yannas, and L.J. Gibson, Prog. Mater. Sci., 46, 273 (2001).

24. H. Sawalha, K. Schroen, and R. Boom, J. Appl. Polym. Sci., 104, 959 (2007).

25. R.A. Zoppi, S. Contant, E.A.R. Duek, F.R. Marques, M.L.F. Wada, and S.P. Nunes, Polymer, 40, 3275 (1999).
26. L. Soletti, Y. Hong, J. Guan, J.J. Stankus, M.S. El-Kurdi, W.R. Wagner, and D.A. Vorp, Acta Biomater., 6, 110 (2010).

27. A. Nieponice, L. Soletti, J. Guan, B.M. Deasy, J. Huard, W.R. Wagner, and D.A. Vorp, Biomaterials, 29, 825 (2008).

28. J.D. Roh, G.N. Nelson, M.P. Brennan, T.L. Mirensky, T. Yi, T.F. Hazlett, G. Tellides, A.J. Sinusas, J.S. Pober, W.M. Saltzman, T.R. Kyriakides, and C.K. Breuer, Biomaterials, 29, 1454 (2008).

29. Y. Song, J.W.H. Wennink, M.M.J. Kamphuis, I. Vermes, A.A. Poot, J. Feijen, and D.W. Grijpma, J. Biomed. Mater. Res. A, 95, 440 (2010).

30. S. Siau, A. Vervaet, S. Degrande, E. Schacht, and A. Van Calster, Appl. Surf. Sci., 245, 353 (2005).

31. B.B. Bird, W.E. Stewart, and E.N. Lightfoot, Transport Phenomena, Wiley, New York (2002). 